\title{
Cycling hypoxia increases U87 glioma cell radioresistance via ROS induced higher and long-term HIF-1 signal transduction activity
}

\author{
CHIA-HUNG HSIEH ${ }^{1}$, CHENG-HUNG LEE ${ }^{1}$, JI-AN LIANG $^{2}$, CHUN-YEN YU $^{2}$ and WOEI-CHERNG SHYU ${ }^{3}$ \\ ${ }^{1}$ Graduate Institute of Basic Medical Science, ${ }^{2}$ Department of Radiation Therapy and Oncology and \\ ${ }^{3}$ Department of Neurology, Center for Neuropsychiatry, and Graduate Institute of Immunology, \\ China Medical University and Hospital, Taichung, Taiwan, R.O.C.
}

Received June 21, 2010; Accepted August 27, 2010

DOI: $10.3892 /$ or_00001027

\begin{abstract}
Glioblastoma multiforme (GBM) tumors are the most common type of brain tumors and resistance to radiotherapy. This study aimed to investigate the differential effect and mechanism of tumor microenvironments, cycling hypoxia and non-interrupted hypoxia, on tumor cell radiosensitivity in the human U87 glioblastoma tumor model. We exposed U87 cells and mice bearing U87 glioma to experimentally imposed cycling or non-interrupted hypoxic stress in vitro and in vivo prior to treatment with ionizing irradiation. Clonogenic survival assay and tumor growth measurements were performed to determine tumor radiosensitivity. The differential regulation of non-interrupted vs. cycling hypoxia by hypoxia-inducible factor-1 (HIF-1) and the impact of HIF-1 $\alpha$ on hypoxia-induced radioresistance were assessed by molecular assay and RNAiknockdown technology. Our results demonstrated that cycling hypoxia induced higher and longer term HIF-1 signal transduction activity via reactive oxygen species (ROS) in U87 cells compared with non-interrupted hypoxia. Cycling hypoxiainduced HIF- $1 \alpha$ activation reflected ROS mediated HIF- $1 \alpha$ synthesis and stabilization, whereas non-interrupted hypoxiainduced HIF- $1 \alpha$ activation was due to decreased HIF- $1 \alpha$ degradation resulting from decreased prolyl hydroxylation. With regard to tumor radiosensitivity, cycling hypoxia induced more tumor cell radioresistance and a decreased response to radiotherapy in U87 cells compared with non-interrupted hypoxia. HIF-1 knockdown during in vitro and in vivo hypoxic stresses combined with radiotherapy suppressed cycling and non-interrupted hypoxia-induced radioresistance
\end{abstract}

Correspondence to: Dr Chia-Hung Hsieh, Graduate Institute of Basic Medical Science, China Medical University and Hospital, No. 91, Hsueh-Shih Road, Taichung 404, Taiwan, R.O.C.

E-mail: chhsiehcmu@mail.cmu.edu.tw

Key words: glioblastoma multiforme, cycling hypoxia, noninterrupted hypoxia, hypoxia-inducible factor-1, reactive oxygen species while increasing overall tumor radiosensitivity. Our results suggest that cycling hypoxia induces more radioresistance than non-interrupted hypoxia in U87 gliomas, and ROS mediated HIF-1 $\alpha$ activation is a crucial mechanism involved in hypoxia-induced differential radioresistant in U87 gliomas.

\section{Introduction}

Glioblastoma multiforme (GBM) is the most common and aggressive form of brain tumor (1). Current therapy consists of surgical resection followed by radiation therapy and concomitant chemotherapy. Despite these treatments, the prognosis for patients is poor (2). Radiotherapy is ideally suited to the treatment of intracranial lesions because it is not limited by drug targeting and can conform to highly irregular target volumes. However, this treatment remains a challenge for radiation oncologists because of tumor radioresistance. The intrinsic radiosensitivity of gliomas is a pivotal factor that determines the effectiveness of radiotherapy (3). Many factors, including genetic alterations and microenvironments, affect tumor response to ionizing radiation (4). One such factor is hypoxia. Because the efficacy of ionizing radiation relies directly on adequate oxygen tensions, tumor hypoxia is a major constraint in tumor treatment by radiotherapy.

Tumor hypoxia has been classified into two models (5). The classic model of hypoxia is that of chronic hypoxia, which is caused by diffusion limitation. A second type of hypoxia, acute hypoxia, is formed by the blood vessels within a tumor have intermittent blood flow due to their abnormal structure or function (6). Since cycling changes in vessel perfusion lead to rapid changes in tumor oxygenation, it is also referred to as 'cycling hypoxia' or 'intermittent hypoxia'. It is thought that chronic hypoxia, rather than acute hypoxia, plays the main role in promotion of cancer progression and in the efficacy of radiation therapy or chemotherapy. This is because the major phenotypic shift associated with chronic hypoxia involves tumor cell resistance to chemotherapy or radiotherapy in addition to more invasive and metastatic features (7). However, this concept contradicts previous findings that tumor regrowth after radiation therapy tended to occur more frequently in regions where there was a high incidence of intermittent vascular stasis (8). Therefore, 
cyclically hypoxic cells might be the most radioresistant cells and could therefore be responsible for tumor regrowth.

There is ample evidence to suggest that cycling hypoxia as well as chronic hypoxia play roles in many aspects of tumor development and growth (9-11). In vitro and in vivo studies have shown that cycling hypoxia can modulate tumor growth, angiogenesis, invasion and metastasis in several tumor models. These complex effects may be regulated by hypoxia-inducible factor-1 (HIF-1). Although the detailed mechanism is complex and still unclear, recent studies have shown that cycling hypoxia can promote HIF-1 stabilization and increase HIF-1 transcriptional activity (9). HIF-1 has been referred to as a critical transcription factor that, in hypoxia, drives the induction or repression of a myriad of genes controlling multiple cell functions such as angiogenesis, metabolism, invasion, metastasis, apoptosis and therapy resistance $(12,13)$. For that reason, HIF-1 may be a key mediator involved in cycling hypoxia-mediated tumor radiosensitivity in GBM.

In the present study, we determined a direct causal link between cycling hypoxia and tumor radiosensitivity in GBM and documented the differential effects of non-interrupted vs. cycling hypoxia in this process. Here, we show that cycling hypoxia induces more radioresistance than non-interrupted hypoxia in U87 gliomas, and ROS mediated HIF-1 $\alpha$ activation is a crucial mechanism involved in hypoxia-induced differential radioresistant in U87 gliomas.

\section{Materials and methods}

Cell culture. U87 cells were cultured in Eagle's minimal essential medium (Life Technologies) supplemented with $10 \%$ fetal bovine serum (FBS), $10 \mathrm{mM}$ HEPES and $1 \%$ penicillin-streptomycin. Cells were maintained at $37^{\circ} \mathrm{C}$ in a humidified incubator containing $5 \% \mathrm{CO}_{2}$ and $20 \% \mathrm{O}_{2}$ in air.

In vitro hypoxic treatments. The cells were treated in Biospherix C-Chamber (Biospherix) inside a standard culture chamber by means of exhausting and gassing with $95 \% \mathrm{~N}_{2}$ and $5 \% \mathrm{CO}_{2}$ to produce oxygen concentrations of $0.5-1 \%$ for $4 \mathrm{~h}$ at $37^{\circ} \mathrm{C}$ to achieve chronic hypoxic condition. For cycling hypoxic treatments, cell cultures were exposed to 3 cycles consisting of $0.5-1 \% \mathrm{O}_{2}$ for $1 \mathrm{~h}$ interrupted by $5 \% \mathrm{CO}_{2}$ and air for $30 \mathrm{~min}$ at $37^{\circ} \mathrm{C}$ in hypoxia chamber by timer-controlled regulator.

ROS production assay. ROS production was determined by incubating U87 cells in the presence of $10 \mu \mathrm{M}$ H2DCFDA under non-interrupted hypoxia, cycling hypoxia or normoxia. Fluorescence was measured in a SpectraMax M2/M2e Microplate Readers (Molecular Devices) with excitation at $485 \mathrm{~nm}$ and emission at $520 \mathrm{~nm}$.

Real-time quantitative PCR. The q-PCR analysis was prepared as described previously (14). The primers used in this quantitative analysis of HIF-1 $\alpha$, VEGF and the housekeeping gene $60 \mathrm{~S}$ acidic ribosomal protein were: for HIF- $1 \alpha$ were (F) 5'-TCCAGTTACGTTCCTTCGATCA-3' and (R) 5'-TTTGAGGACTTGCGCTTTCA-3'; for VEGF, 5'-CAGC TACTGCCATCCAATCGA-3' and 5'-CTGGCCTTGGTGA
GGTTTGAT-3'; for the house-keeping gene 60S acidic ribosomal protein (F) 5'-ACGAGGTGTGCAAGGAGGGC-3' and (R) 5'-GCAAGTCGTCTCCCATCTGC-3'.

Western blot analysis. Cells were lysed and nuclear extracts were prepared as described previously (14). HIF-1 $\alpha$ protein in human cells was detected in $150 \mu \mathrm{g}$ of cell extract or nuclear extract using monoclonal HIF-1 $\alpha$ antibody (1:750; Novus) and HIF-1 $\alpha$ hydroxyl P564 antibody (1:1000; Novus). Western blots were normalized using a monoclonal anti- $\beta$ actin antibody (diluted 1:10000; Santa Cruz Technology).

Plasmid constructs. The lentiviral vector pLKO AS2 (National RNAi Core Facility, Taiwan) was used as the backbone to generate inducible HIF-responsive firefly luciferase construct. The multiple cloning sites (MCS) of pTA-Luc vector (Clontech) was inserted with the cDNA fragment bearing eight repeats of the hypoxia-responsible element (HRE) from the 3' enhancer region of human Epo gene to drive the expression of firefly luciferase gene. The HIF-1-inducible reporter gene cassette was amplified from 8 X HRE to SV40 ploy A on the constructed pTA-Luc vector using PCR and inserted into pLKO AS2 by XhoI and $M l u I$ restriction enzymes. Furthermore, the lentiviral vector pLVCT-tTRKRAB (Addgene) was used to express HIF- $1 \alpha$ shRNA or non-targeting control sequence (Sigma) following the manufacturer's protocol.

Lentivirus production and infection. Lentiviruses were produced as described elsewhere (15). U87 cells were infected at various MOI and the infection-efficiency curve at $72 \mathrm{~h}$ was determined by counting EYFP positive cells. More than $90 \%$ of infected cells were obtained at 100 MOI for U87 cells after $72 \mathrm{~h}$.

Small interfering RNA transfection. U87 cells were transfected with different small interfering RNA (siRNA): SiScramble (5'-ACUCUAUCUGCACGCUGACUU-3'), and siRNA specific for HIF-1 $\alpha$ (5'-UUCAAGUUGGAAUUGG UAG-3'). Cells were transfected with $20 \mathrm{nmol} / \mathrm{l}$ of the different siRNA (SiScramble and SiHIF-1 $\alpha$ ) using the Oligofectamine transfection reagent following the instructions provided by the manufacturer.

Luciferase assays. Luciferase activity was determined by mixing $10 \mu \mathrm{l}$ of extracts from $1 \times 10^{5}$ cells and $100 \mu \mathrm{l}$ of luciferase assay reagent (Promega). Light production was measured for $10 \mathrm{sec}$ in a SpectraMax M2/M2e Microplate Readers (Molecular Devices).

Flow cytometry analysis. The percentage of cells expressing EYFP and the intensity of expression were analyzed by a flow cytometer (BD FACSCanto), and the data were processed with BD FACSDvia software. Histograms of EYFP were plotted with the log scale for EYFP fluorescence and the results were recorded as means of the middle of the peaks.

Cell irradiation. Cultured control or hypoxia pretreated cells were transported on ice, and then were irradiated $(2.85 \mathrm{~Gy} /$ $\mathrm{min}$ ) under normoxic conditions at room temperature using a 
high-energy X-ray linear accelerator (Varian). Cells were then harvested at appropriate time-points for in vitro clonogenic survival assay.

In vitro clonogenic survival assay. Exponentially growing cells were trypsinized, counted and diluted accordingly. These cell suspensions were then irradiated at $4 \mathrm{~Gy}$ and plated in $60-\mathrm{mm}$ dishes in triplicate. After 14 days, colonies were fixed and stained with crystal violet in $50 \%$ ethanol. Colonies consisting of $>50$ cells were counted to determine surviving fractions.

In vivo hypoxic treatments. The procedure for in vivo hypoxic treatments was carried out following published methods $(10,11)$. Briefly, the tumor-bearing mice were exposed to a continuous flow of a humidified gas mixture to induce in vivo hypoxia in the 6-1 hypoxia chambers. The mice were exposed to either normal air for control group or $7 \% \mathrm{O}_{2}$ for $4 \mathrm{~h}$ for chronic hypoxia treatment or 3 cycles of $1 \mathrm{~h} 7 \% \mathrm{O}_{2}$ breathing interrupted by $30 \mathrm{~min}$ periods of normal air breathing for cycling hypoxia treatment. Tumor oxygen was determined using the Oxford Oxylite fiberoptic probe (Oxford).

Immunohistochemistry. The tumor tissues were fixed in $4 \%$ paraformaldehyde and embedded in OCT compound. Five micrometer sections were immunostained with mouse monoclonal anti-HIF-1 $\alpha$ (Novus Biologicals), visualized with an AEC kit (InnoGenex) and counterstained with hematoxylin.

Animal irradiation. The $2 \times 10^{6}$ U87 cells with conditional knockdown of HIF-1 $\alpha$ were injected in mouse flanks. The cells were allowed to establish tumors for 2 weeks postinjection and then were treated with or without $3 \mathrm{ml}$ of doxycycline $(500 \mathrm{~g} / \mathrm{ml})$ intraperitoneally following in vivo hypoxic treatments before irradiation. At $1 \mathrm{~h}$ after hypoxic treatments, tumors in mice were locally irradiated (4.25 $\mathrm{Gy} / \mathrm{min}$ ) at a dose of $10 \mathrm{~Gy}$ under anesthesia using a highenergy X-ray linear accelerator (Varian).

Growth delay studies. Tumor size was measured with calipers every day for up to 14 days after irradiation. Tumor volume was calculated from caliper measurements of length and width of masses: volume $\left(\mathrm{mm}^{3}\right)=$ length $\mathrm{x}$ width ${ }^{2} \times 1 / 2$ and expressed as cubic millimeters.

In vivo/in vitro clonogenic survival assay. Tumors were then excised, minced and dissociated for $30 \mathrm{~min}$ at $37^{\circ} \mathrm{C}$ in Hanks' balanced salt solution containing $166 \mathrm{U} / \mathrm{ml}$ collagenase XI, $0.25 \mathrm{mg} / \mathrm{ml}$ protease and $225 \mathrm{U} / \mathrm{ml}$ DNase. Cells were recovered by straining through an $80 \mu \mathrm{m}$ mesh and centrifuging at $500 \mathrm{~g}$ and were resuspended in culture medium. Cells were counted by hemocytometer using trypan blue and assessed by in vitro clonogenic survival assay.

Statistical analysis. For multiple comparisons, the KruskalWallis ANOVA was used for non-parametric variables. For parametric variables, ANOVA was used along with Fisher's least-significant-difference (LSD). $\mathrm{P}<0.05$ was considered significant. All analyses were two-tailed.

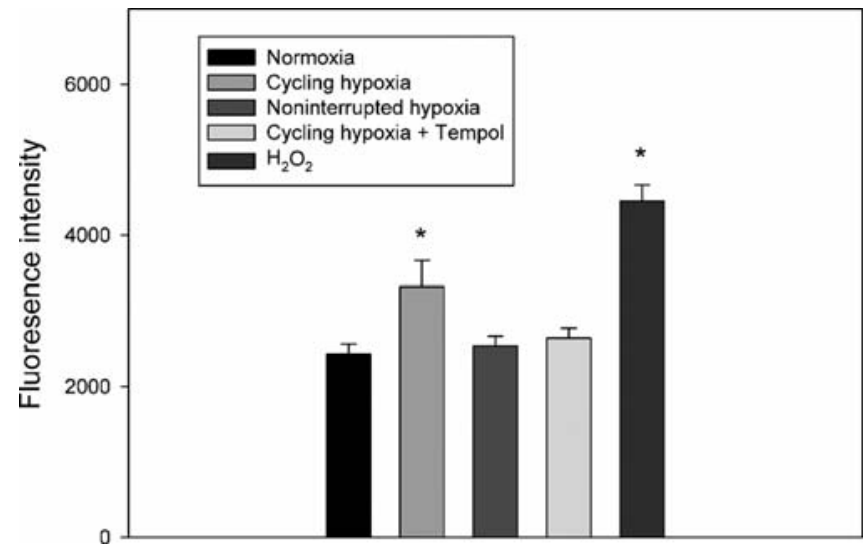

Figure 1. Effect of non-interrupted hypoxia and cycling hypoxia on ROS production in U87 cells. Cells were treated with in vitro non-interrupted or cycling hypoxic stress for $4 \mathrm{~h}$ in the absence or presence of $300 \mu \mathrm{g} / \mathrm{ml}$ Tempol, and the level of intracellular peroxide was evaluated by a DCHF-DA assay via flow cytometric analysis. Each bar represents the mean \pm SD calculated from three independent experiments. *Significantly different from control at $\mathrm{P}<0.05$.

\section{Results}

Effects of non-interrupted hypoxia and cycling hypoxia on ROS production in U87 glioma cells. A series of reoxygenation phases interrupting the hypoxia periods under cycling hypoxia may lead to ROS production in the living cells. Therefore, we first examined the effect of experimentally imposed non-interrupted or cycling hypoxic stress on ROS production. U87 cells were exposed to in vitro hypoxic treatments and ROS production was determined using the H2DCF-DA probe together with FACS. We observed an increase in fluorescent signal in cycling hypoxia treated cells (Fig. 1). In contrast to cycling hypoxia treated cells, nonsignificant increase in ROS formation was found in normoxic or non-interrupted hypoxic cells. Furthermore, Tempol, a membrane-permeable radical scavenger, could eliminate cycling hypoxia-induced ROS. These results indicate that cycling hypoxia triggered ROS production in U87 glioma cells.

Effects of non-interrupted hypoxia and cycling hypoxia on HIF-1a synthesis and stabilization in U87 glioma cells. We examined the effect of experimentally imposed non-interrupted or cycling hypoxic stress on HIF-1 regulation. The amounts of HIF-1 $\alpha$ protein in nuclear extracts were assayed by Western blot analysis after $4 \mathrm{~h}$ of in vitro hypoxic treatment. Under both non-interrupted and cycling hypoxic stress, U87 cells experienced an increase in HIF- $1 \alpha$ protein levels. HIF- $1 \alpha$ protein levels in U87 cells under cycling hypoxia stress were higher than in U87 cells under non-interrupted hypoxia stress (Fig. 2A). Moreover, the result from real-time PCR analysis, as shown in Fig. 2B, demonstrates that HIF-1 $\alpha$ was also increased, exhibiting a maximal 1.5- to 1.8-fold increase after $4 \mathrm{~h}$ of non-interrupted or cycling hypoxic treatment compared with normoxia treatment. The mRNA expression levels in the cycling hypoxia-treated group were slightly higher than in the non-interrupted hypoxia-treated group. Tempol inhibited the increase in HIF-1 $\alpha$ protein and mRNA levels induced by cycling hypoxia. 


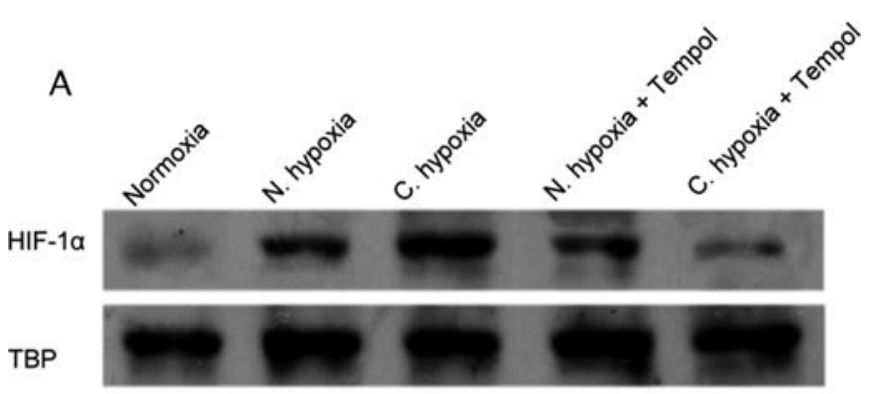

N. hypoxia= noninterrupted hypoxia C. hypoxia $=$ cycling hypoxia

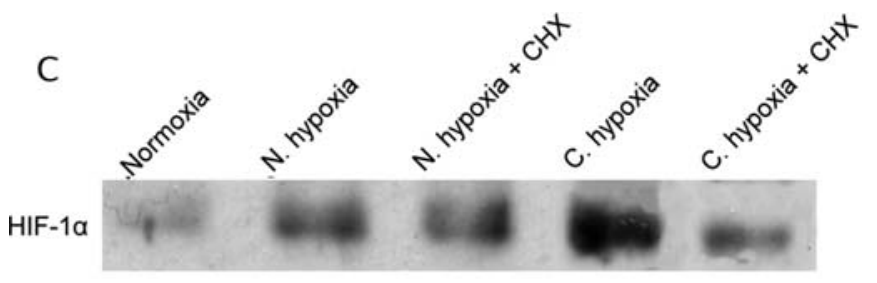

$\beta$-actin

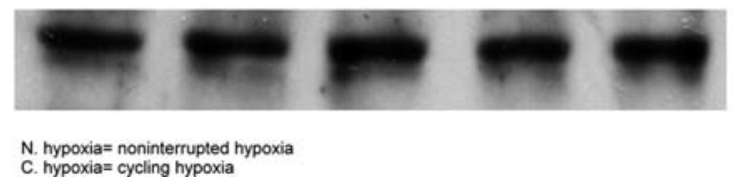

B

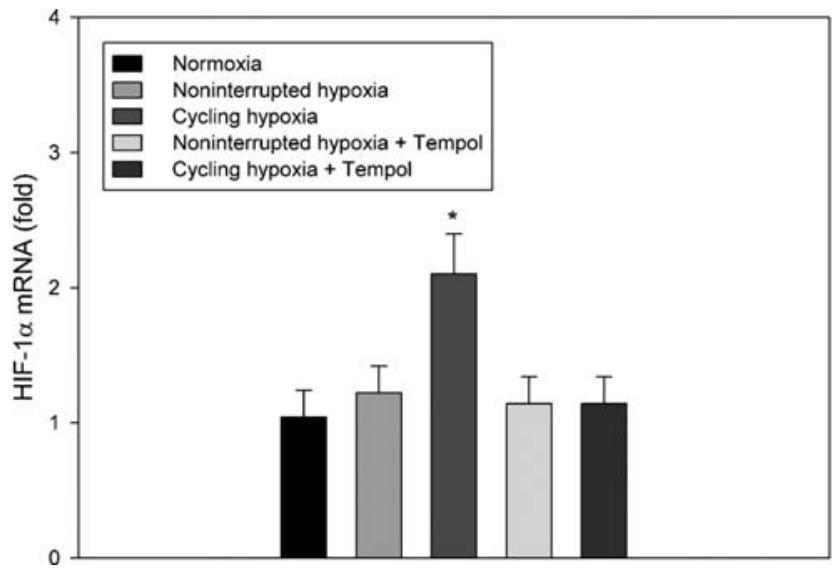

D

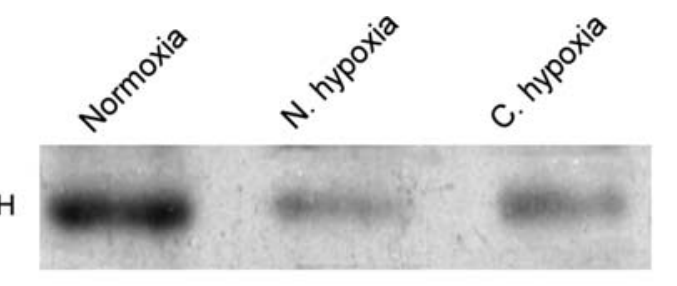

$\beta$-actin

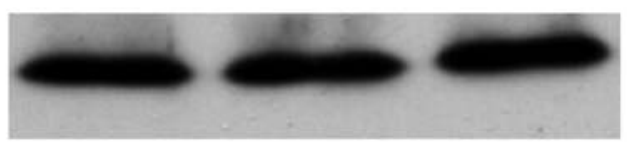

N. hypoxia $=$ noninterrupted hypoxia C. hypoxia $=$ cycling hypoxia

Figure 2. Effect of non-interrupted hypoxia and cycling hypoxia on HIF-1 $\alpha$ synthesis and stabilization in U87 cells. (A) Western blot analysis of HIF-1 $\alpha$ in U87 cells after non-interrupted hypoxia and cycling hypoxia. (B) Real-time PCR analysis of HIF-1a in cells after hypoxic stresses described in the previous panel. HIF- $1 \alpha$ mRNA levels are relative to the expression of the $60 \mathrm{~S}$ acidic ribosomal protein reference gene. Error bars denote the standard deviation within triplicates. "Significantly different from control at P<0.05. (C) Western blot analysis of HIF-1 $\alpha$ in cells with or without cycloheximide (CHX) followed by hypoxic stresses. (D) Western blot analysis of HIF-1 $\alpha$ hydroxylation in cells after hypoxic stresses.

Next, glioma cells were treated with cycloheximide $(50 \mu \mathrm{g} /$ $\mathrm{ml}$ ) followed by exposure to non-interrupted or cycling hypoxia. Our results demonstrated that cells exposed to cycling hypoxia with cycloheximide experienced less HIF-1 $\alpha$ accumulation than those exposed to cycling hypoxia without cycloheximide (Fig. 2C). The HIF-1 $\alpha$ levels in cells exposed to non-interrupted hypoxia with cycloheximide were similar to those of cells exposed to non-interrupted hypoxia without cycloheximide. This result suggests that cycling hypoxiainduced HIF-1 $\alpha$ accumulation was due, at least in part, to new protein synthesis. Moreover, tumor hypoxia inhibition of HIF- $1 \alpha$ prolyl hydroxylases led to decreased HIF-1 $\alpha$ hydroxylation and degradation. We then examined whether cycling hypoxia affected HIF-1 $\alpha$ hydroxylation using an antibody specific to the hydroxylated form of HIF- $1 \alpha$ (HIF- $1 \alpha-\mathrm{OH})$. As shown in Fig. 2D, both cycling and non-interrupted hypoxia-induced HIF-1 $\alpha$ accumulations were associated with a marked decrease in HIF- $1 \alpha-\mathrm{OH}$ levels. This indicated that both cycling and non-interrupted hypoxia could induce HIF-1 $\alpha$ stabilization. Taken together, these results suggest that cycling hypoxia-induced HIF- $1 \alpha$ accumulation reflects increased HIF-1 $\alpha$ synthesis and stabilization, whereas noninterrupted hypoxia-induced HIF- $1 \alpha$ accumulation is due to decreased HIF-1 $\alpha$ degradation resulting from decreased prolyl hydroxylation. ROS are crucial mediators involved in cycling hypoxia mediated HIF-1 activation.

Effects of non-interrupted hypoxia and cycling hypoxia on HIF-1 signal transduction activity in U87 glioma cells. As demonstrated above, hypoxic stresses cause differential expression of HIF-1 $\alpha$ in U87 cells. We next verified whether this effect could further induce differential HIF-1 signal transduction activity. To achieve this, U87 cells were stably transfected with a lentiviral vector bearing a HIF-1-inducible luciferase for monitoring the activity of HIF-regulated signal transduction pathways in cultured cells. As shown in Fig. 3A, HRE transcriptional activity in the cycling hypoxia-treated cells was significantly increased after treatment, with maximal activation occurring after $48 \mathrm{~h}$. HRE transcriptional activity subsequently decreased to low levels at $72 \mathrm{~h}$ after treatment. In contrast to cycling hypoxia-treated cells, HRE transcriptional activity in the non-interrupted hypoxia-treated cells was rapidly induced, peaking within $24 \mathrm{~h}$ after treatment and then gradually declining to low levels at $48 \mathrm{~h}$ posttreatment. Moreover, we also determined mRNA expression of $V E G F$, a HIF-1 downstream target gene, at various times after hypoxic treatments. The VEGF mRNA expression profiles obtained after exposure to cycling or non-interrupted hypoxia were 

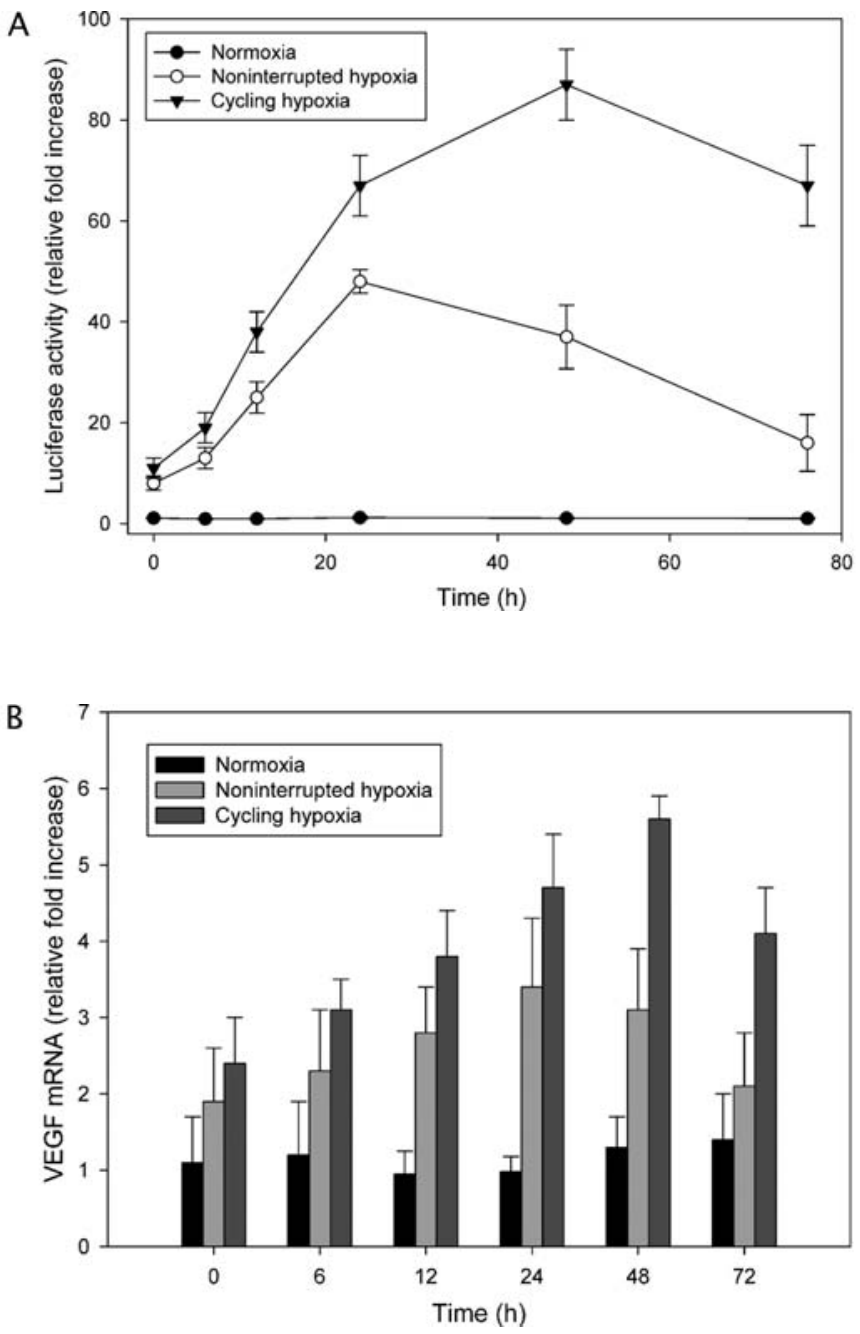

Figure 3. Effects of non-interrupted hypoxia and cycling hypoxia on HIF-1 signal transduction activity in U87 cells. (A) Transcriptional activity of the hypoxia response elements in U87 cells after non-interrupted or cycling hypoxic stress. Error bars show standard deviation of triplicate experiments. (B) VEGF mRNA expression in U87 cells after non-interrupted or cycling hypoxic stress. VEGF mRNA levels are relative to the expression of the $60 \mathrm{~S}$ acidic ribosomal protein reference gene. Error bars denote the standard deviation within triplicates

similar (Fig. 3B), suggesting that cycling hypoxia could induce and prolong higher HIF-1 signal transduction activity than could hypoxia in response to higher HIF-1 $\alpha$ protein accumulation in cell nuclei.

Effects of non-interrupted hypoxia and cycling hypoxia on cell viability and radiosensitivity in U87 glioma cells. Next, we investigated the effects of experimentally imposed hypoxic stress, either non-interrupted or cycling, on radiosensitivity. We used the clonogenic survival assay to differentiate the effects of non-interrupted hypoxia and cycling hypoxia on the response of U87 cells to single doses of 4-Gy ionizing radiation. Our results showed that both noninterrupted and cycling hypoxia pretreatment significantly increased cell resistance to ionizing radiation compared with normoxic controls in U87 cells (Fig. 4A). The surviving cell fraction of U87 cells preconditioned by the application of cycling hypoxic stress followed by irradiation was significantly higher than those preconditioned by the application

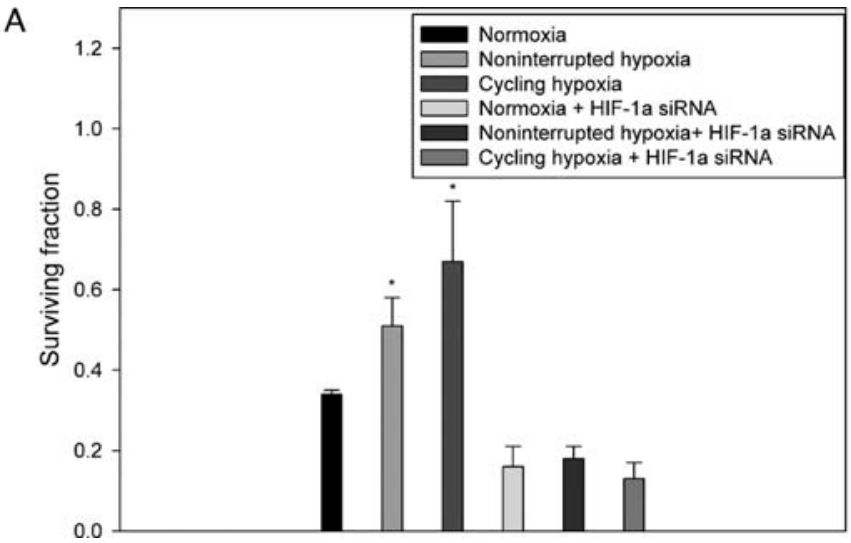

B

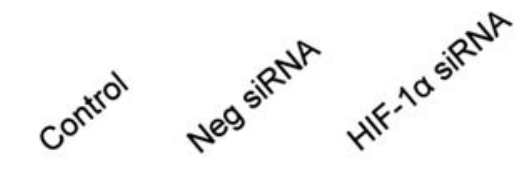

HIF-1a

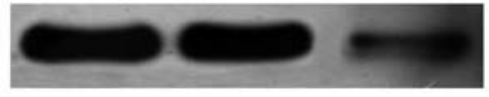

$\beta$-actin

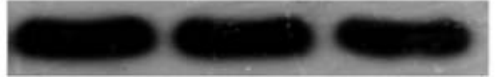

Figure 4. Effects of non-interrupted hypoxia and cycling hypoxia on cell viability and radiosensitivity in U87 cells. (A) Clonogenic survival assay of U87 cells with or with HIF-1 $\alpha$ siRNA after $4 \mathrm{~h}$ of non-interrupted or cycling hypoxic stress followed by 4-Gy irradiation. Error bars denote the standard deviation within triplicates. *Significantly different from control at $\mathrm{P}<0.05$. (B) Western blot analysis of siRNA-mediated down-regulation of HIF-1 $\alpha$ in U87 cells under hypoxia for $18 \mathrm{~h}$.

of non-interrupted hypoxic stress followed by irradiation. Cycling hypoxic stress was capable of producing more radioresistant U87 cells than non-interrupted hypoxic stress. Finally, we determined whether the transcription factor HIF-1 $\alpha$ was a crucial mediator involved in hypoxia-induced radioresistance in U87 cells. We used a specific silencing small interfering RNA (siRNA) to knock down HIF-1 $\alpha$ induction in U87 cells under hypoxic stress. This siRNA successfully knocked down HIF-1 $\alpha$ expression, whereas the negative control (Neg) siRNAs did not (Fig. 4B). When HIF-1 $\alpha$ induction was blocked by siRNA, the radioresistance induced by non-interrupted or cycling hypoxic stress was lost (Fig. 4A). These results indicated that cycling hypoxia could induce more radioresistance than non-interrupted hypoxia in U87 cells. Thus, HIF-1 $\alpha$ was shown to be a crucial mediator involved in hypoxia-induced radioresistance in U87 cells.

Effects of non-interrupted hypoxia and cycling hypoxia on tumor radiosensitivity in U87 glioma xenografts. To determine the effects of non-interrupted or cycling hypoxic stress on tumor response to radiotherapy and to determine whether HIF- $1 \alpha$ is a crucial mediator involved in hypoxia-induced radioresistance in U87 glioma xenografts, we first utilized Tet-regulatable lentiviral vectors encoding short hairpin RNAs (shRNAs) to stably and specifically knock down HIF-1 $\alpha$ in U87 cells. These cells expressed a low level of HIF-1 $\alpha$ in the presence of doxycycline under hypoxic conditions (Fig. 5A). In addition, we injected these cells into the flanks of mice 


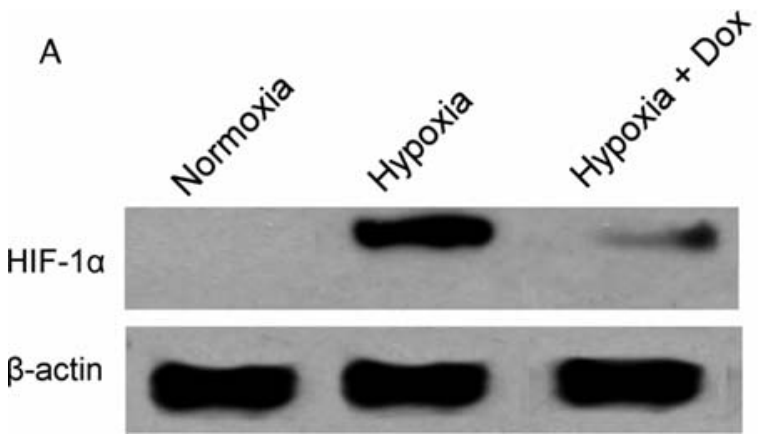

B

\section{Normoxia}

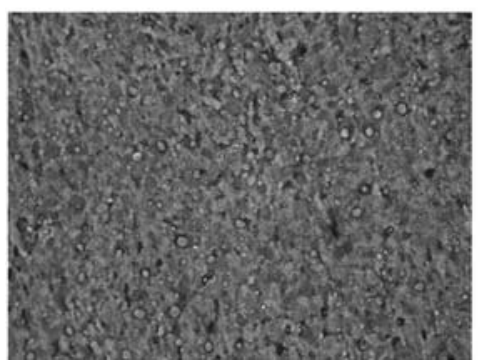

Hypoxia

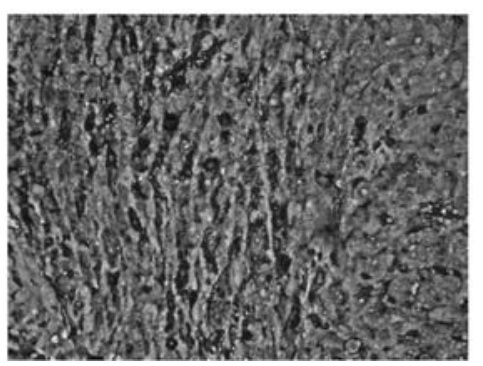

Hypoxia + Dox

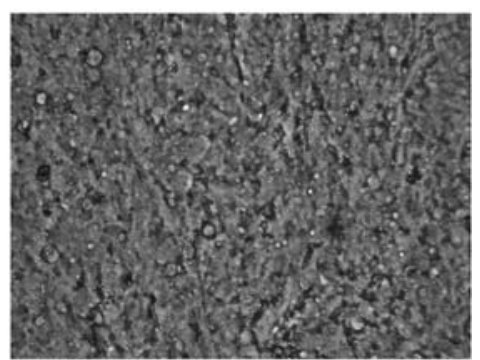

C

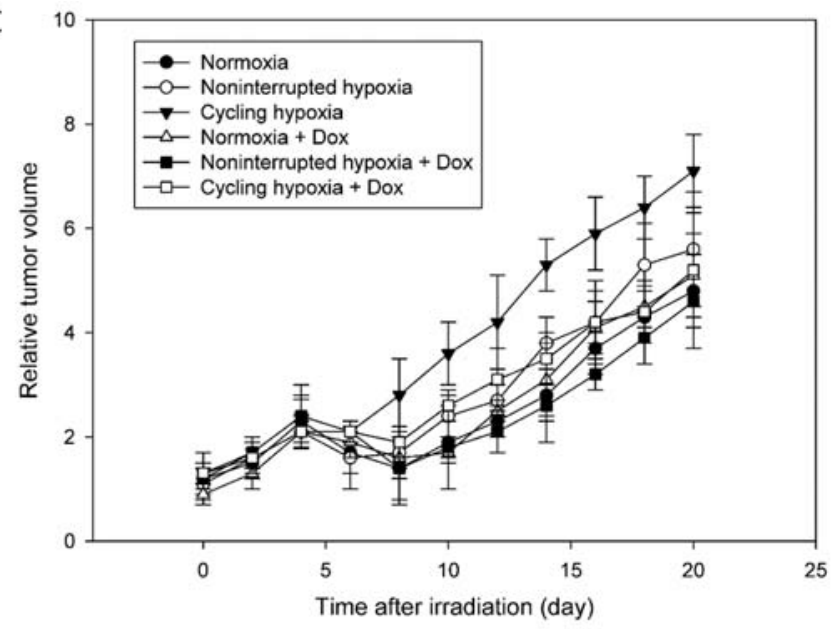

D

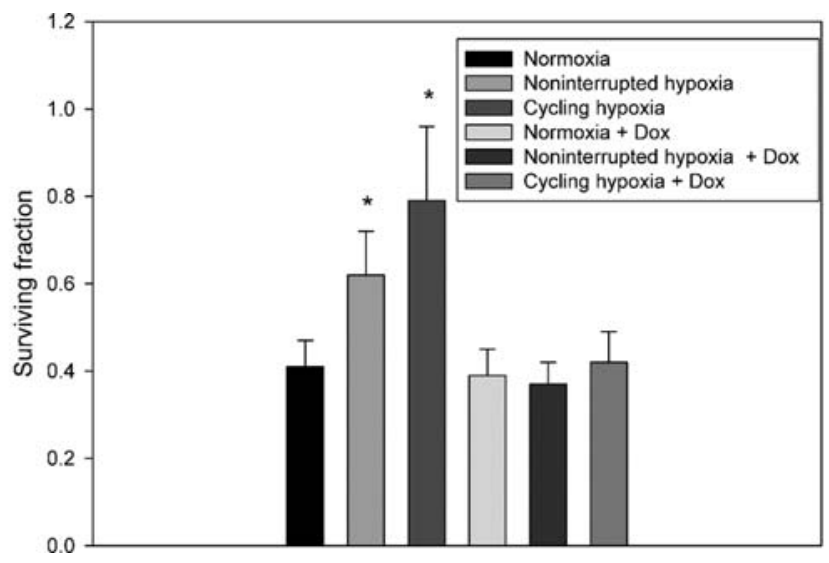

Figure 5. Effects of non-interrupted hypoxia and cycling hypoxia on tumor radiosensitivity in U87 glioma xenografts. (A) Western blot analysis of HIF-1 $\alpha$ regulation by using doxycycline-inducible shRNA in U87 cells under hypoxia for $18 \mathrm{~h}$. (B) Immunohistochemical analysis of HIF-1 $\alpha$ in U87 glioma xenografts with or without conditional knockdown of HIF-1 $\alpha$ under hypoxic stress. Original magnification, x200. (C) Tumor growth delay in U87 glioma xenografts. The data represent the means \pm standard deviation of tumor volumes from 8 to 10 mice. (D) Surviving fraction in excised tumors after irradiation as described in panel (C). Error bars represent the standard deviation within triplicates. "Significantly different from control at $\mathrm{P}<0.05$.

and 14 days postinjection tumors had developed. Mice were then treated with $3 \mathrm{ml}$ of doxycycline $(500 \mathrm{~g} / \mathrm{ml})$ or control, intraperitoneally (i.p.) following in vivo hypoxia treatment. The results from HIF- $1 \alpha$ immunohistochemical analysis demonstrated that doxycycline induction of RNAi targeting HIF-1 $\alpha$ in vivo also led to suppression of HIF-1 $\alpha$ expression in U87 tumors under hypoxic conditions (Fig. 5B). The tumor-bearing mice were treated with or without doxycycline following in vivo hypoxia treatment before irradiation. At $1 \mathrm{~h}$ after in vivo hypoxic treatments, mice received local tumor irradiation at a dose of $10 \mathrm{~Gy}$, and tumor volumes were measured on subsequent days. As shown in Fig. 5C, specific growth delay was significantly less in cycling hypoxiapretreated U87 tumors compared with non-interrupted hypoxia-pretreated U87 tumors or U87 control tumors. However, knockdown of HIF-1 $\alpha$ in U87 human glioblastoma xenografts under hypoxic stress resulted in the inhibition of non-interrupted or cycling hypoxic stress-induced radioresistance.

To further analyze the effect of non-interrupted or cycling hypoxic stress on xenograft radiosensitivity, we performed tumor cell colony formation assays to quantify tumor cell clonogenic potential after cycling or non-interrupted hypoxic stress in U87 xenografts. The clonogenicity of tumors was compared after irradiation of non-interrupted hypoxiapretreated mice, cycling hypoxia-pretreated mice and control mice. We observed a significant increase in cell surviving fraction in cells isolated from U87 xenografts in hypoxic 
stress-treated mice (either non-interrupted or cycling hypoxia) following 10-Gy irradiation compared with cells isolated from U87 xenografts under normoxia following 10-Gy irradiation (Fig. 5D). However, the surviving cell fraction after cycling hypoxic stress followed by irradiation was significantly higher than that in non-interrupted hypoxic stress followed by irradiation. In addition, knockdown of HIF-1 $\alpha$ in U87 xenografts under non-interrupted or cycling hypoxic stress resulted in a significant reduction in clonogenicity after 10-Gy irradiation. Taken together, these data contrast with our in vitro growth results and suggest that cycling hypoxia could induce more radioresistance than noninterrupted hypoxia and that hypoxia-induced HIF-1 activation is a vital determinant of tumor radiosensitivity.

\section{Discussion}

Radiobiologists or radiation oncologists have known for many years that hypoxia renders cells radioresistant (16). However, these studies focused mainly on the effects of chronic hypoxia on radiosensitivity. These previous studies clearly demonstrated that chronic hypoxia protects tumor cells from apoptosis induced by radiotherapy and chemotherapy and that it increases tumor cell resistance for these treatments $(17,18)$. In recent years, studies have pointed out that the effects of cycling hypoxia on cancer progression are different from those of chronic hypoxia. Cycling hypoxia has been shown to render tumors more invasive in animal models $(10,11)$. Moreover, cycling hypoxia positively modulates angiogenic processes and radioresistance in the vascular endothelium of tumors (9). We report here that both noninterrupted and cycling hypoxia pretreatment significantly increases cell resistance to ionizing radiation compared with normoxic controls in U87 cells or U87 glioma xenografts. Moreover, we found that cycling hypoxia treatment had a greater effect on increased radiation resistance compared with non-interrupted hypoxia treatment. Consequently, cycling hypoxia induced more radioresistance than noninterrupted hypoxia in our experiments. Thus, our results are to verify the hypothesis derived from the earlier findings of Yamaura and Matsuzawa that cycling-hypoxic cells might be the most radioresistant and could therefore be responsible for tumor regrowth (8). Our findings strongly support the notion that cycling hypoxia can exert different effects than those induced by chronic hypoxia (19).

Typically, hypoxic tumors are resistant to radiotherapy and the majority of conventional chemotherapeutic agents (20). A critical mediator of hypoxia-induced tumor resistance to radiotherapy and chemotherapy is HIF-1 $\alpha$, which upregulates expression of antiapoptotic proteins, such as IAP-2 or BCL-xL, that promote antiapoptosis or mediate expression of VEGF and bFGF in tumor cells, thereby encouraging endothelial cell survival $(21,22)$. HIF-1 activity is deregulated in many human cancers, especially those that occur under hypoxic conditions or that are the result of genetic alterations (23). In the past, the effects of chronic hypoxia on HIF-1 regulation have been extensively studied, and it has been clearly demonstrated that chronic hypoxia can stabilize HIF-1 $\alpha$ due to blockage of the degradation pathway, further inducing its signal transduction activity (13). It has been shown that cells exposed to cycling hypoxia exhibit a more robust HIF-1 response than cells that are chronically hypoxic (9). The results from earlier works, as well as our present results, clearly demonstrate that cells exposed to cycling hypoxia can induce more HIF- $1 \alpha$ protein expression and activity than that observed under chronic hypoxia. Moreover, our results further indicate that the mechanisms involving HIF-1 $\alpha$ under chronic or cycling hypoxia are different. Cycling hypoxia-induced HIF-1 $\alpha$ protein expression reflects increased HIF-1 $\alpha$ synthesis and stabilization, whereas the increase in chronic hypoxia-induced HIF- $1 \alpha$ protein expression is due to decreased HIF-1 $\alpha$ degradation resulting from reduced prolyl hydroxylation. ROS are crucial mediators involved in cycling hypoxia mediated HIF-1 activation. These findings are important in the selection of optimal genetic or pharmacological approaches for anticancer therapy targeting HIF-1 (23). Combining inhibitors of HIF-1 protein synthesis with activators of HIF-1 protein degradation may be more effective in suppressing HIF-1 protein accumulation induced by tumor hypoxia.

GBM tumors contain multiple hypoxic areas that exhibit elevated HIF-1 signal transduction activity. This results in the increased expression of many downstream target genes that contribute to GBM tumor malignancy (24). In this study, we used a direct experimental technique to modify tumor oxygenation and induce additional non-interrupted or cycling hypoxia in tumors. By using such an approach, we can directly observe the effects of non-interrupted and cycling hypoxia on relevant responses or mechanisms in living subjects. Our data demonstrate that a smaller growth delay and higher surviving fraction in response to irradiation were observed in the cycling hypoxia-pretreated U87 tumors compared with non-interrupted hypoxia-pretreated U87 tumors or U87 control tumors. Based on these results, hypoxia-induced HIF-1 activation may be a vital determinant of tumor radiosensitivity. Indeed, conditional knockdown of HIF-1 $\alpha$ in U87 glioma xenografts during in vivo hypoxic treatments and the subsequent receipt of radiotherapy restored the effects of tumor growth delay and tumor cell survival, demonstrating the importance of HIF-1 $\alpha$ in hypoxiainduced radioresistance. Therefore, inhibition of HIF-1 activity could represent an important component of combination radiotherapy to enhance tumor radiosensitivity.

In summary, the study presented here takes a significant step toward understanding the differing mechanisms by which chronic hypoxia and cycling hypoxia effect tumor radiosensitivity. Moreover, our data highlight the importance of cycling hypoxia on tumor cell radiosensitivity. Thus, radiobiologists and oncologists should be aware of cycling hypoxiainduced radioresistance in cancer treatment. Furthermore, our results suggest that HIF-1 blockade should be used before irradiation, concurrent with radiotherapy, and should be maintained throughout the course of radiotherapy to maximally increase overall tumor radiosensitivity.

\section{Acknowledgements}

This study was supported by grant 97-2314-B-039-033-MY3 from the National Science Council, Taipei, Taiwan, and grants CMU97-221 and CMU98-BC-02 from China Medical University, Taichung, Taiwan. 


\section{References}

1. Sulman EP, Guerrero $M$ and Aldape K: Beyond grade: molecular pathology of malignant gliomas. Semin Radiat Onco 19: 142-149, 2009.

2. Salgaller ML and Liau LM: Current status of clinical trials for glioblastoma. Rev Recent Clin Trials 1: 265-281, 2006.

3. Noda SE, El-Jawahri A, Patel D, Lautenschlaeger T, Siedow M and Chakravarti A: Molecular advances of brain tumors in radiation oncology. Semin Radiat Oncol 19: 171-178, 2009.

4. Vaupel P: Tumor microenvironmental physiology and its implications for radiation oncology. Semin Radiat Oncol 14: 198-206, 2004

5. Coleman CN: Hypoxia in tumors: a paradigm for the approach to biochemical and physiologic heterogeneity. J Natl Cancer Inst 80: 310-317, 1988 .

6. Durand RE: Intermittent blood flow in solid tumours - an underappreciated source of 'drug resistance'. Cancer Metastasis Rev 20: 57-61, 2001

7. Kirkpatrick JP, Cardenas-Navia LI and Dewhirst MW: Predicting the effect of temporal variations in $\mathrm{PO} 2$ on tumor radiosensitivity. Int J Radiat Oncol Biol Phys 59: 822-833, 2004.

8. Yamaura $\mathrm{H}$ and Matsuzawa T: Tumor regrowth after irradiation; an experimental approach. Int J Radiat Biol Relat Stud Phys Chem Med 35: 201-219, 1979.

9. Martinive P, Defresne F, Bouzin C, et al: Preconditioning of the tumor vasculature and tumor cells by intermittent hypoxia: implications for anticancer therapies. Cancer Res 66: 11736-11744, 2006.

10. Cairns RA and Hill RP: Acute hypoxia enhances spontaneous lymph node metastasis in an orthotopic murine model of human cervical carcinoma. Cancer Res 64: 2054-2061, 2004.

11. Cairns RA, Kalliomaki T and Hill RP: Acute (cyclic) hypoxia enhances spontaneous metastasis of KHT murine tumors. Cancer Res 61: 8903-8908, 2001.

12. Semenza GL: HIF-1 and tumor progression: pathophysiology and therapeutics. Trends Mol Med 8: S62-S67, 2002.
13. Harris AL: Hypoxia - a key regulatory factor in tumour growth. Nat Rev Cancer 2: 38-47, 2002.

14. Hsieh CH, Kuo JW, Lee YJ, Chang CW, Gelovani JG and Liu RS: Construction of mutant TKGFP for real-time imaging of temporal dynamics of HIF-1 signal transduction activity mediated by hypoxia and reoxygenation in tumors in living mice. J Nucl Med 50: 2049-2057, 2009.

15. Szulc J and Aebischer P: Conditional gene expression and knockdown using lentivirus vectors encoding shRNA. Methods Mol Biol 434: 291-309, 2008.

16. Hockel M, Schlenger K, Mitze M, Schaffer U and Vaupel P: Hypoxia and radiation response in human tumors. Semin Radiat Oncol 6: 3-9, 1996.

17. Haensgen G, Krause U, Becker A, et al: Tumor hypoxia, p53, and prognosis in cervical cancers. Int J Radiat Oncol Biol Phys 50: 865-872, 2001 .

18. Saito Y, Milross CG, Hittelman WN, et al: Effect of radiation and paclitaxel on p53 expression in murine tumors sensitive or resistant to apoptosis induction. Int J Radiat Oncol Biol Phys 38: 623-631, 1997.

19. Dewhirst MW: Relationships between cycling hypoxia, HIF-1, angiogenesis and oxidative stress. Radiat Res 172: 653-665, 2009.

20. Unruh A, Ressel A, Mohamed HG, et al: The hypoxia-inducible factor-1 alpha is a negative factor for tumor therapy. Oncogene 22: 3213-3220, 2003.

21. Greijer AE and van der Wall E: The role of hypoxia inducible factor 1 (HIF-1) in hypoxia induced apoptosis. J Clin Pathol 57: 1009-1014, 2004.

22. Semenza GL: Intratumoral hypoxia, radiation resistance, and HIF-1. Cancer Cell 5: 405-406, 2004.

23. Semenza GL: Targeting HIF-1 for cancer therapy. Nat Rev Cancer 3: 721-732, 2003.

24. Oliver L, Olivier C, Marhuenda FB, Campone M and Vallette FM: Hypoxia and the malignant glioma microenvironment: regulation and implications for therapy. Curr Mol Pharmacol 2: 263-284, 2009. 